\title{
Special Issue of Hematopoietic Stem Cell Transplantation
}

\section{Porrata Luis $\mathrm{F}^{*}$}

Luis F. Porrata, MD, Division of Hematology, Mayo Clinic, Rochester, MN, USA and Member of the Editorial Board of Journal of Stem Cell Research \& Therapy, USA

In this special issue of Hematopoietic Stem Cell Transplantation we will provide comprehensive reviews by experts in the field of hematopoietic stem cell transplantation that cover a range of topics from basic science, translational research and clinical practice.

Leinge et al. [1] present a study comparing flow cytometry (FC) and quantitative real time polymerase chain reaction (RT-qPCR) assay to enumerate the number of $\mathrm{CD} 34+/ \mathrm{CD} 61+$ megakaryocytic progenitors collected during apheresis and infused to patients undergoing hematopoietic stem cell transplantation. Despite the fact that both FC and RT-qPCR were able to quantify a subset of CD34+/CD61+ cells, the enumeration of this subset did not correlate with platelet recovery post-hematopoietic stem cell transplantation. Leinge's study provides a needed platform to build upon the development of better tests for the quantification of stem cell progenitors to eliminate the serious complication of engraftment failure as it has been reported that delayed platelet recovery at day 100 after allogeneic stem cell transplantation is associated with survival.

Shiksha et al. [2] provide an up-today review of one of the most common and potentially serious side effects observed in hematopoietic stem cell transplantation: infection complications. This is a succinct but comprehensive review of the most common bacterial, parasitic, fungal, and viral infections encountered by stem cell transplantation clinicians. Table 1 depicts the risk of early and late infections depending on the type of stem cell transplantations: autologous stem cell transplantation and the different varieties of allogeneic stem cell transplantation including syngeneic, matched-related, matched-unrelated, haploidentical, cordblood, and nonmyeloablative. As more frequent and serious infection complications are observed in the allogeneic stem cell transplantation in comparison to autologous stem cell transplantation, figure 1 of this review diagramed a schematic representation of the pre-engraftment, post-engraftment, and late phase specific opportunistic infections experienced by patients undergoing allogeneic stem cell transplantation. Thus, this review provides stem cell transplant clinicians with an uptoday, quick, easy access reference to identify and manage infection complications during the course and long-term follow-up of patients treated with hematopoietic stem cell transplantation.

Hartwig [3] writes a provocative review of the potential use of telomerase and tumor suppression biology to engineer healthy hematopoietic stem cells to treat patients with stem cell transplantation. As stem cell transplantation patients relied on the infusion of healthy stem cells to engraft and sustain the hematopoietic compartment cell turnover of approximately $10^{9}$ cells produced per hour, Hartwig reviews two mechanisms associated with hematopoietic stem cell senesces, apoptosis, and exhaustion: telomerase shortening and DNA damage accumulation. The review points out the association between DNA damage accumulation and telomerase activity as marker of cancer development and the role of telomerase activity as the most common mechanism of replicative immortality. Therefore, for the development of strategies to engineer long-lasting, healthy hematopoietic stem cells needs to address the possibility of cancer development by the mechanisms of DNA damage accumulation and telomerase shortening. Hartwig describe a two-step strategy for hematopoietic stem cells in vitro expansion to engineer healthy long-lasting hematopoietic stem cells. The two-step procedure includes culturing hematopoietic stem cells under up-regulation of telomerase activity to promote longstanding self-renewal capabilities and tumor suppression responses to prevent or reduce DNA damage accumulation along cell divisions. This technology not only could apply to generation of healthy long-lasting self-renewal hematopoietic stem cells for stem cell transplantation, but also to use these engineered hematopoietic stem cells for regenerative medicine for the differentiation of other cells types such as new myocytes to treat myocardial infarction or new neurons for the treatment of Parkinson disease.

Powers and Trobridge [4] have written a state of the art review discussing the use of retroviral mutagenesis for hematopoietic stem cell gene therapy by analyzing virus insertion sties to identify novel engraftment genes to enhance our understanding of the engraftment process post-hematopoietic stem cell transplantation and to serve as a platform to develop therapeutic interventions to improve engraftment and prevent the serious deleterious consequence of graft failure.One of such interventions as described by Powers and Trobridge is the transduction of harvested hematopoietic stem cells with retroviral vectors providing proviral integration sites that confer an engraftment advantage once these modified hematopoietic stem cells are infused back to patients undergoing hematopoietic stem cell transplantation.

In collaboration with my colleague Kerryn Ansell [5], we reviewed the role of myeloid-derived suppressor cells (MDSC) affecting the benefits of an autologous graft versus tumor effect in patients treated with autologous stem cell transplantion. These monocytic MDSC are cells that are collected and re-infused to patients undergoing autologous stem cell transplant. This significant findings provides a new therapeutic intervention of an autograft monocytic depletion to minimize or abrogate the infusion of these immunosuppressive cells; thus, enhancing host immune recovery and immunity that has been published and confirmed to improve clinical outcomes in patients undergoing autologous stem cell transplantation.

Each article in this special issue of Hematopoietic Stem Cell Transplantation was authored by investigators who played a leading role in the advances described. I am confident the this Hematopoietic Stem Cell Transplantation special issue will be a resource for all investigators in the field, as well as clinical practitioners in hematopoietic stem cell transplantation and laboratory scientist in related fields.

*Corresponding author: Luis F. Porrata, MD, Division of Hematology, Mayo Clinic, Rochester, MN, USA, Tel. 507-284-3158; Fax: 507-266-4972; E-mail: porrata.luis@mayo.edu

Received October 16, 2013; Accepted October 18, 2013; Published October 21, 2013

Citation: Porrata Luis F (2013) Special Issue of Hematopoietic Stem Cell Transplantation. J Stem Cell Res Ther S3: e001. doi:10.4172/2157-7633.S3-e001

Copyright: (C) 2013 Porrata Luis F. This is an open-access article distributed under the terms of the Creative Commons Attribution License, which permits unrestricted use, distribution, and reproduction in any medium, provided the original author and source are credited. 
Citation: Porrata Luis F (2013) Special Issue of Hematopoietic Stem Cell Transplantation. J Stem Cell Res Ther S3: e001. doi:10.4172/2157-7633. S3-e001

\section{References}

1. Leinge E, Nielsen KR, Baech J, Steffensen R, Dybkaer K, et al. (2011) Quantitation of Megakaryocytic Progenitors in Apheresis Products by Flow Cytometry and Real Time PCR. J Stem Cell Res Ther S3-001.

2. Shiksha K, Pranab SA, Farhan M, Huy N, Deepk A, et al. (2013) Infectious Complications of Hematopoietic Stem Cell Transplantation. J Stem Cell Res Ther S3-002.

3. Hartwig FP (2013) Up-Regulating Telomerase and Tumor Suppression: A Two-
Step Strategy to Boost Hematopoietic Stem Cell Transplantation. J Stem Cell Res Ther S3-003.

4. Powers JM, Trobridge GD (2013) Identification of Hematopoietic Stem Cell Engraftment Genes in Gene Therapy Studies. J Stem Cell Res Ther S3-004.

5. Ansell K Porrata LF (2013) Autograft Monocyte" The Bad Humors of Autologous Peripheral Blood Hematopoietic Stem Cell Transplantation. J Stem Cell Res Ther S3-005.

This article was originally published in a special issue, Hematopoietic stem cell transplantation handled by Editor(s). Dr. Porrata Luis F, Mayo Clinic Transplant Center, USA 